International Journal of Pure and Applied Mathematics

Volume 115 No. 3 2017, 641-650

ISSN: 1311-8080 (printed version); ISSN: 1314-3395 (on-line version)

url: http://www.ijpam.eu

doi: 10.12732/ijpam.v115i3.16

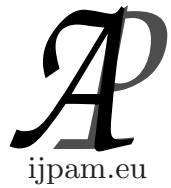

\title{
SOME INVERSE RESULTS ABOUT MATRIX STABILITY IN TERMS OF MATRIX MEASURE
}

\author{
Hristo Kiskinov $^{1}$, Nedelcho Milev $^{2}$, Andrey Zahariev ${ }^{3}$ \\ ${ }^{1,2,3}$ Faculty of Mathematics and Informatics \\ University of Plovdiv \\ 4 Tzar Asen, 4000 Plovdiv, BULGARIA
}

\begin{abstract}
The aim of the present work is for each complex matrix $A \in \mathbb{C}^{n \times n}$ with spectral abscissa $S(A)<0$ and for each vector norm $\|$.$\| in \mathbb{C}^{n}$ to establish an explicit construction to obtain a matrix measure $\mu_{\|.\|}$with $\mu_{\|.\|}(A)<0$. Furthermore the proved result is extended for the case when the entries $a_{k}^{j}$ of the matrix $A$ are continuous functions, i.e. $a_{k}^{j} \in C(K, \mathbb{C})$, where $K \subset \overline{\mathbb{C}}_{+}$is an arbitrary compact set.
\end{abstract}

AMS Subject Classification: 15A60, 37C75, 65F35

Key Words: matrix measure, logarithmic norm, Lozinskii measure, logarithmic efficiency, logarithmically optimal norm and matrix measure

\section{Introduction}

Many mathematical facts about matrix stability are playing an important role for numerous concrete applications as useful tools for practical calculation on the base of which we can make important qualitative conclusions. For example it is well known that if a square matrix $A=\left\{a_{k}^{j}\right\}_{k, j=1}^{n} \in \mathbb{C}^{n \times n}$ is stable (i.e. all eigenvalues of have negative real parts) then the zero solution of the corresponding first order (or fractional order $\alpha \in(0,1)$ ) linear autonomous system of differential equations is globally asymptotical stable. An explicit suf-

Received: July 14, 2017

Revised: June 10, 2017

Published: July 27, 2017

${ }^{\S}$ Correspondence author (c) 2017 Academic Publications, Ltd. url: www.acadpubl.eu 
ficient condition which guaranties that the eigenvalues have negative real parts is $\mu(A)<0$, where $\mu: \mathbb{C}^{n \times n} \rightarrow \mathbb{R}$ is an arbitrary matrix measure (also known as logarithmic norm or Losinskii measure). Unfortunately it is not difficult to create an example which shows that the inverse statement is generally not true, i.e. all eigenvalues of the matrix can have negative real parts but it is possible that there exists a matrix measure with $\mu(A) \geq 0$. This fact leads to great difficulties in the proofs of several results from hereditary and comparison type.

An inverse result is proved from Zahreddine in [10] (Theorem 2.11). The statement of this theorem is that $S(A)=\inf _{\|.\| \in \Upsilon} \mu_{\|.\|}(A)$, where $S(A)$ denotes the spectral abscissa of the matrix $A, \Upsilon$ denotes the set of all possible vector norms in $\mathbb{C}^{n}$ and with $\mu_{\|.\|}(A)$ is denoted the corresponding matrix measure induced from an arbitrary norm $\|.\| \in \Upsilon$. This work was the first motivation to extend and improve the results obtained in it for practical application. The main consequence of the proved result in [10] is that if $S(A)<0$ then there exists at least one matrix measure $\mu_{\|.\|}$with $\mu_{\|.\|}(A)<0$. It is an elegant mathematical result, but from point of view of the applications it is very difficult (maybe impossible) to calculate the infimum in this relation over all possible vector norms in $\mathbb{C}^{n \times n}$, which decrease essentially the practical application of this result. More detailed, this result cannot help us to find practically some matrix measure $\mu_{\|.\|}$with $\mu_{\| .||}(A)<0$ or for a concrete norm used in some proof to conclude that for the matrix measure $\mu_{\|.\|}$induced from this norm, the inequality $\mu_{\|.\|}(A)<0$ holds. These are the main reasons for our concept for each matrix $A \in \mathbb{C}^{n \times n}$ with spectral abscissa $S(A)<0$ and for each vector norm $\|$.$\| in \mathbb{C}^{n}$ to introduce an explicit construction under which we obtain a matrix measure $\mu_{\|.\|}$with $\mu_{\| .||}(A)<0$.

The paper is organized as follows. In Section 2, we recall some needed definitions and some results about the matrix measure (named also logarithmic norm [1] or Lozinskii measure [5]) for square complex matrices. Section 3 is devoted to our main results about the matrix measure for complex matrices. We prove that for each complex matrix $A \in \mathbb{C}^{n \times n}$ with spectral abscissa $S(A)<0$ and for each vector norm $\|$.$\| in \mathbb{C}^{n}$ can be explicitly constructed a matrix measure $\mu_{\|.\|}$with $\mu_{\|.\|}(A)<0$. Furthermore the proved result is extended for the case when the entries $a_{k}^{j}$ of the matrix $A$ are continuous functions, i.e. $a_{k}^{j} \in C(k, \mathbb{C})$, where $K \subset \overline{\mathbb{C}}_{+}$is an arbitrary compact set. The last mentioned result can be used for example in the stability analysis of the neutral linear fractional differential systems even with distributed delays.

Note that our considerations are related with the stability analysis as well as with estimation of eigenvalue range for complex interval and parametric 
matrices. For more details we refer the works of O. Pastravanu at all $[6,7,8]$, L. Kolev at all $[2,3,4]$ and the references therein.

\section{Preliminaries}

In this section to avoid possible misunderstandings, we recall some definitions and results about the matrix measure (named also logarithmic norm or Lozinskii measure) for complex matrices.

Let denote $\mathbb{C}_{+}=\{p \in \mathbb{C} \mid \operatorname{Rep}>0\}, \overline{\mathbb{C}}_{+}=\{p \in \mathbb{C} \mid \operatorname{Rep} \geq 0\}, \mathbb{C}_{-}=\mathbb{C} \backslash \overline{\mathbb{C}}_{+}$. For every matrix $A=\left\{a_{k}^{j}\right\}_{k, j=1}^{n} \in \mathbb{C}^{n \times n}$ we denote with $A^{T}=\left\{a_{j}^{k}\right\}_{k, j=1}^{n}, \bar{A}=$ $\left\{\bar{a}_{k}^{j}\right\}_{k, j=1}^{n}$ and $A^{*}=\left\{\bar{a}_{j}^{k}\right\}_{k, j=1}^{n}$ the transpose, the conjugate and the Hermitian transpose of $A$ respectively. With $S p(A)$ we denote the spectrum of $A, \rho(A)=$ $\max _{\lambda \in S p(A)}|\lambda|$ is the spectral radii and $S(A)=\sup \{\operatorname{Re} \lambda \mid \lambda \in S p(A)\}$ the spectral abscissa of $A$.

Let denote with $\Upsilon$ the set of all possible vector norms in $\mathbb{C}^{n}$ and let $\|.\| \in \Upsilon$ be an arbitrary norm. For $H \in \mathbb{C}^{n \times n}$ we define the induced matrix norm in $\mathbb{C}^{n \times n}$ as usual $\|H\|=\max _{z \in \mathbb{C}^{n},\|z\|=1}\|H z\|$.

Definition 1. ([1], [5]) For an arbitrary norm $\|.\| \in \Upsilon$ the corresponding matrix measure is defined by

$$
\mu(A)=\lim _{\eta \rightarrow+0} \frac{\|I+\eta A\|-1}{\eta}
$$

for each matrix $A \in \mathbb{C}^{n \times n}$.

Note that if a matrix norm $\|$.$\| is not induced by some vector norm in \mathbb{C}^{n}$ (as described above), then for the existence of the limit in Definition 1 the matrix norm must satisfy two additional conditions: $\|I\|=1$ and $\|A B\| \leq\|A\|\|B\|$ for each $A, B \in \mathbb{C}^{n \times n}$ where $I$ is the identity-matrix.

Using an arbitrary norm $\|.\| \in \Upsilon$ as generating norm, we define the corresponding family of weighted norms as follow:

$$
W_{\|.\|}^{H}=\left\{\|z\|^{H}=\|H z\| \mid H \in \mathbb{C}^{n \times n}, \operatorname{det} H \neq 0, z \in \mathbb{C}^{n}\right\}
$$

Then for every matrix $A=\left\{a_{k}^{j}\right\}_{k, j=1}^{n} \in \mathbb{C}^{n \times n}$ the corresponding induced matrix norm is $\|A\|^{H}=\left\|H A H^{-1}\right\|$ and the corresponding induced matrix measure is $\mu^{H}(A)=\mu\left(H A H^{-1}\right)$.

For every $A, B \in \mathbb{C}^{n \times n}, \alpha \geq 0$, and $p \in \mathbb{C}$ the following relations hold: 
(a) $\mu(\alpha A+p I)=\alpha \mu(A)+R e p$

(b) $-\|A\| \leq-\mu(-A) \leq \mu(A) \leq\|A\|$

(c) $\mu(A)+\mu(-A) \geq 0$

(d) $\mu(A+B) \leq \mu(A)+\mu(B)$

(e) $-\mu(-A) \leq \operatorname{Re} \lambda \leq \mu(A)$ for each $\lambda \in S p(A)$

In the partial case for the Holder vector $q$-norm defined by $\|z\|_{q}=\left(\sum_{i=1}^{n}\left|z_{i}\right|^{q}\right)^{\frac{1}{q}}$, $\|z\|_{\infty}=\max _{1 \leq i \leq n}\left\{\left|z_{i}\right|\right\}$, the corresponding matrix measure can be calculated explicitly in the cases :

(a) $\mu_{1}(A)=\sup _{1 \leq j \leq n}\left\{\operatorname{Re} a_{j}^{j}+\sum_{k=1, k \neq j}^{n}\left|a_{k}^{j}\right|\right\}$, for $q=1$,

(b) $\mu_{2}(A)=\frac{1}{2} S\left(A+A^{*}\right)$, for $q=2$,

(c) $\mu_{\infty}(A)=\sup _{1 \leq k \leq n}\left\{\operatorname{Re} a_{k}^{k}+\sum_{j=1, j \neq k}^{n}\left|a_{k}^{j}\right|\right\}$, for $q=\infty$.

Definition 2. ([9]) The logarithmic inefficiency of an arbitrary norm $\|.\| \in \Upsilon$ with respect to the matrix $A$ is given by $q(A)=\mu(A)-S(A)$, where $\mu(A)$ is the corresponding induced matrix measure.

Definition 3. ([9]) A norm and the corresponding matrix measure are logarithmically optimal with respect to $A$ if $q(A)=0$ and logarithmically $\epsilon$ efficient if for some $\epsilon>0$ we have $q(A) \leq \epsilon$.

\section{Main Results}

The statement of the next theorem is a practically applicable generalization of Theorem 2.11 in [10]. As a generating norm we will take an arbitrary norm $\|.\| \in \Upsilon$, but in the practice as generating norms are used the mostly applicable norms as the Holder's vector $q$-norm, $q \in \mathbb{N} \cup\{\infty\}$.

Theorem 4. Let $A=\left\{a_{k}^{j}\right\}_{k, j=1}^{n} \in \mathbb{C}^{n \times n}$ be an arbitrary stable matrix, i.e. $S(A)<0,\|.\| \in \Upsilon$ be an arbitrary norm and $\epsilon>0$ be an arbitrary number.

Then there exists $\delta^{*}=\delta^{*}(\epsilon, A)>0$ such that for each $\delta \in\left(0, \delta^{*}\right)$ there exists a matrix $H_{\delta} \in \mathbb{C}^{n \times n}$, det $H_{\delta} \neq 0$, for which the corresponding induced matrix 
measure $\mu^{H_{\delta}}$ is logarithmically $\epsilon$-efficient with respect to $A$, i.e. $\mu^{H_{\delta}}(A)-$ $S(A) \leq \epsilon$.

Proof. Let $A=\left\{a_{k}^{j}\right\}_{k, j=1}^{n} \in \mathbb{C}^{n \times n}$ be an arbitrary stable matrix, i.e. $S(A)<$ 0 and $\|.\| \in \Upsilon$ be an arbitrary norm. Then according Jordan's theorem there exists a nonsingular matrix $H \in \mathbb{C}^{n \times n}$, such that $J=H A H^{-1}=\Delta+U$, where $\Delta$ is a diagonal matrix and $U$ is a strictly upper triangular matrix, which offdiagonal entries are the same as the off-diagonal entries of $J$. For each $\delta>0$ and $H \in \mathbb{C}^{n \times n}$ with $\operatorname{det} H \neq 0$ we introduce the matrix $H_{\delta}$ as follows:

$$
H_{\delta}=D_{\delta} H, \quad D_{\delta}=\operatorname{diag}\left(1, \delta^{-1}, \delta^{-2}, \ldots, \delta^{-(n-1)}\right)
$$

Then denoting $y=H_{\delta} z$, for each $\eta>0$ we have

$$
\begin{aligned}
\|I+\eta A\|^{H_{\delta}} & =\max _{\left\|H_{\delta} z\right\|=1}\left\|H_{\delta}(I+\eta A) z\right\|= \\
& =\max _{\|y\|=1}\left\|H_{\delta}(I+\eta A) H_{\delta}^{-1} y\right\|= \\
& =\max _{\|y\|=1}\left\|\left(I+\eta H_{\delta} A H_{\delta}^{-1}\right) y\right\|= \\
& =\max _{\|y\|=1}\left\|\left(I+\eta D_{\delta}(\Delta+U) D_{\delta}^{-1}\right) y\right\|= \\
& =\max _{\|y\|=1}\|(I+\eta \Delta) y+\eta \delta U y\|
\end{aligned}
$$

From (3.2) it follows

$$
\begin{aligned}
\mu^{H_{\delta}}(A) & =\lim _{\eta \rightarrow 0+} \eta^{-1}\left(\|I+\eta A\|^{H_{\delta}}-1\right) \leq \\
& \leq \lim _{\eta \rightarrow 0+} \eta^{-1}(\|I+\eta \Delta\|-1)+\delta\|U\| \leq \mu(\Delta)+\delta\|U\|
\end{aligned}
$$

Then for each $\delta \in\left(0, \epsilon\|U\|^{-1}\right)$ from (3.3) we obtain that

$$
\mu^{H_{\delta}}(A) \leq S(A)+\epsilon
$$

holds for every $\epsilon>0$.

Corollary 5. Let $A=\left\{a_{k}^{j}\right\}_{k, j=1}^{n} \in \mathbb{C}^{n \times n}$ be an arbitrary stable matrix, i.e. $S(A)<0$ and $\|.\| \in \Upsilon$ be an arbitrary norm.

Then the following two assertions hold:

(i) $S(A)=\inf _{\delta>0} \mu^{H_{\delta}}(A)$;

(ii) There exists $\delta^{*}=\delta^{*}(A)>0$, such that $\mu^{H_{\delta}}(A)<0$ for each $\delta \in\left(0, \delta^{*}\right)$. 
Proof. (i) Taking into account that (3.4) holds for each $\epsilon>0$, then the assertion (i) follows immediately from (3.4).

(ii) Since $S(A)<0$ then the assertion (ii) follows from (3.4) for every $\epsilon \in\left(0,2^{-1}|S(A)|\right.$.

Remark 6. Theorem 2.11 in [10] claims $S(A)=\inf _{\|.\| \in \Upsilon} \mu_{\|.\|}(A)$, where with $\mu_{\|. \mid\|}(A)$ is denoted the corresponding matrix measure induced from an arbitrary norm $\|.\| \in \Upsilon$. But it must be noted, that in [10] the result is proved only for Euclidian norm $\|.\|_{2}$ as generating norm, which in our opinion makes the proof there incomplete.

Remark 7. The main advantage of Theorem 4 and Corollary 5 in compare with Theorem 2.11 in [10] is that we can take every practically used norm as generating norm and construct the corresponding family of weighted norms. Then we can take the infimum only over the constructed family of weighted norms (which obviously can be different for the different generating norms) instead of calculating infimum over all possible norms, which can be very difficult or maybe impossible.

Theorem 4 and Corollary 5 provide several possibilities to obtain results concerning stability for example of fractional differential systems with delays. But as a shortcoming of the obtained results we can point its "locality". Usually the entries of the characteristic matrix of the (fractional) differential or functional- differential systems with delays are continuous (even entire) functions of complex variable $A(p)=\left\{a_{k}^{j}(p)\right\}_{k, j=1}^{n}, p \in \mathbb{C}$, i.e. in general the entries are not constants. Then there is no guarantee that if for some $p_{*} \in \mathbb{C}$ the corresponding induced matrix measure $\mu^{H_{\delta}}$ is logarithmically $\epsilon$-efficient with respect to $A\left(p_{*}\right)$, the same matrix measure will be logarithmically $\epsilon$-efficient with respect to $A(p)$ for some other $p \neq p_{*}$. The next theorem is an important step to overcome this obstacle.

Theorem 8. Let $A, B \in \mathbb{C}^{n \times n}$ be arbitrary stable matrices, $\|.\| \in \Upsilon$ be an arbitrary norm and $\epsilon>0$ be an arbitrary number.

Then there exists $\delta^{*}=\delta^{*}(\epsilon, A, B)>0$ such that for each $\delta \in\left(0, \delta^{*}\right)$ there exists a matrix $H_{\delta} \in \mathbb{C}^{n \times n}$, $\operatorname{det} H_{\delta} \neq 0$ defined by (3.1), for which the corresponding induced matrix measure $\mu^{H_{\delta}}$ is logarithmically $\epsilon$-efficient with respect to $A$ and $B$ together, i.e. $\mu^{H_{\delta}}(A)-S(A) \leq \epsilon$ and $\mu^{H_{\delta}}(B)-S(B) \leq \epsilon$.

Proof. Let $A, B \in \mathbb{C}^{n \times n}$ be arbitrary stable matrices, i.e. $S(A)<0, S(B)<$ 0 and let $\|.\| \in \Upsilon$ be an arbitrary norm and $\epsilon>0$ be an arbitrary number. According Jordan's theorem there exist nonsingular matrices $H, Q \in \mathbb{C}^{n \times n}$, 
such that $J=H A H^{-1}=\Delta+U, B=Q J_{*} Q^{-1}=Q\left(\Delta_{*}+U_{*}\right) Q^{-1}$, where $\Delta$ and $\Delta_{*}$ are diagonal matrices, $U$ and $U_{*}$ are strictly upper triangular matrices, which off-diagonal entries are the same as the off-diagonal entries of $J$ and $J_{*}$ respectively. Then in virtue of Theorem 4 for each $\delta \in\left(0, \epsilon\|U\|^{-1}\right)$ we have that (3.4) holds, where the matrix $H_{\delta}$ is defined by (3.1). Denoting by $y=H_{\delta} z$ for each $\delta>0$ and $\eta>0$ we have

$$
\begin{aligned}
& \|I+\eta B\|^{H_{\delta}}=\max _{\left\|H_{\delta} z\right\|=1}\left\|H_{\delta}(I+\eta B) z\right\|= \\
& =\max _{\|y\|=1}\left\|H_{\delta}\left(I+\eta Q J_{*} Q^{-1}\right) H_{\delta}^{-1} y\right\|= \\
& =\max _{\|y\|=1}\left\|\left(I+\eta H_{\delta} Q\left(\Delta_{*}+U_{*}\right) Q^{-1} H_{\delta}^{-1}\right) y\right\|= \\
& =\max _{\|y\|=1}\left\|\left(I+\eta(H Q)^{T} D_{\delta}\left(\Delta_{*}+U_{*}\right)(H Q)^{-1} D_{\delta}^{-1}\right) y\right\|= \\
& =\max _{\|y\|=1}\left\|\left(I+\eta(H Q)^{T}\left(\left(D_{\delta} \Delta_{*}\right)+\left(D_{\delta} U_{*}\right)\right)(H Q)^{-1} D_{\delta}^{-1}\right) y\right\|= \\
& =\max _{\|y\|=1}\left\|\left(I+\eta(H Q)^{T}\left(D_{\delta} \Delta_{*}\right)(H Q)^{-1}+\eta(H Q)^{T}\left(D_{\delta} U_{*} D_{\delta}^{-1}\right)\left((H Q)^{T}\right)-1\right) y\right\|= \\
& =\max _{\|y\|=1}\left\|\left(I+\eta\left(\left(D_{\delta} \Delta_{*}\right) H Q\right)(H Q)^{-1} D_{\delta}^{-1}\right) y+\eta \delta(H Q)^{T} U_{*}\left((H Q)^{T}\right)^{-1} y\right\|= \\
& =\max _{\|y\|=1}\left\|\left(I+\eta D_{\delta} \Delta_{*} D_{\delta}^{-1}\right) y+\eta \delta(H Q)^{T} U_{*}\left((H Q)^{T}\right)^{-1} y\right\|= \\
& =\max _{\|y\|=1}\left\|\left(I+\eta \Delta_{*}\right) y+\eta \delta(H Q)^{T} U_{*}\left((H Q)^{T}\right)^{-1} y\right\|
\end{aligned}
$$

From (3.5) it follows

$$
\begin{aligned}
\mu^{H_{\delta}}(B) & =\lim _{\eta \rightarrow 0+} \eta^{-1}\left(\|I+\eta B\|^{H_{\delta}}-1\right) \leq \\
& \leq \lim _{\eta \rightarrow 0+} \eta^{-1}\left(\left\|I+\eta \Delta_{*}\right\|-1\right)+\delta\left\|U_{*}\right\| \leq \\
& \leq \mu\left(\Delta_{*}\right)+\delta\left\|U_{*}\right\|
\end{aligned}
$$

and therefore for each $\delta \in\left(0, \epsilon\left\|U_{*}\right\|^{-1}\right)$ we have that

$$
\mu^{H_{\delta}}(B) \leq S(B)+\epsilon
$$

Then we can conclude that for every $\delta \in\left(0, \epsilon \min \left(\|U\|^{-1},\left\|U_{*}\right\|^{-1}\right)\right)$ both inequalities (3.4) and (3.7) hold. 
Remark 9. Obviously the assertion of Theorem 8 can be extended for an arbitrary finite number $l \in \mathbb{N}$ of matrices. We proved only the case $l=2$ to avoid unnecessarily additional writing.

Theorem 10. Let $\|.\| \in \Upsilon$ be an arbitrary norm, $K \subset \overline{\mathbb{C}_{+}}$be an arbitrary compact set, $A(p)=\left\{a_{k}^{j}(p)\right\}_{k, j=1}^{n}$ and the following conditions hold:

1. The functions $a_{k}^{j} \in C\left(\overline{\mathbb{C}}_{+}, \mathbb{C}\right), 1 \leq k, j \leq n$.

2. $S(A(p))<0$ for every $p \in K$.

Then for each $\epsilon>0$ there exists $\delta^{*}=\delta^{*}(\epsilon,\|\|)>$.0 such that for each $\delta \in\left(0, \delta^{*}\right)$ there exists a matrix $H_{\delta} \in \mathbb{C}^{n \times n}, \operatorname{det} H_{\delta} \neq 0$ defined by (3.1), for which the corresponding induced matrix measure $\mu^{H_{\delta}}$ is logarithmically $\epsilon$-efficient with respect to $A(p)$ for every $p \in K$.

Proof. Let $\|.\| \in \Upsilon$ be an arbitrary norm, $p_{0} \in K$ be an arbitrary fixed point and $H^{0} \in \mathbb{C}^{n \times n}$ be a nonsingular matrix which transforms the matrix $A\left(p_{0}\right)$ in its canonical Jordan form. Let for each $p \in K$ the number $\epsilon_{p} \in\left(0, \min \left(\left|S\left(A\left(p_{0}\right)\right)\right|,|S(A(p))|\right)\right)$ be arbitrary. Then according Theorem 8 there exists $\delta_{p}=\delta_{p}\left(\epsilon_{p},\|\|.\right)>0$ such that for each $\delta \in\left(0, \delta_{p}\right)$ there exists a matrix $H_{\delta_{p}}^{0} \in \mathbb{C}^{n \times n}$ with $\operatorname{det} H_{\delta_{p}}^{0} \neq 0$ for which for the corresponding induced matrix measure $\mu^{H_{\delta_{p}}^{0}}$ both inequalities $\mu^{H_{\delta_{p}}^{0}}\left(A\left(p_{0}\right)\right)<0$ and $\mu^{H_{\delta_{p}}^{0}}(A(p))<0$ hold, where the matrix $H_{\delta_{p}}^{0}$ is defined by $(3.1)$, i.e. $H_{\delta_{p}}^{0}=D_{\delta_{p}} H^{0}$ and $D_{\delta_{p}}=$ $\operatorname{diag}\left(1, \delta_{p}^{-1}, \delta_{p}^{-2}, \ldots, \delta_{p}^{-(n-1)}\right)$. Then condition 1 of Theorem 10 implies that there exists $r\left(p_{0}, p\right)>0$ such that $\mu^{H_{\delta_{p}}^{0}}(A(z))<0$ for every $z \in c_{p_{0}} \cup c_{p}$, where $c_{p}=\left\{z \in \overline{\mathbb{C}}_{+}|| z-p \mid<r\left(p_{0}, p\right)\right\}$ and $c_{p_{0}}=\left\{z \in \overline{\mathbb{C}}_{+}|| z-p_{0} \mid<\right.$ $\left.r\left(p_{0}, p\right)\right\}$. Since the set $c_{p_{0}} \cup\left(\bigcup_{p \in K, p \neq p_{0}} c_{p}\right)$ is an open cover of the compact set $K$ then there exists a finite subcover $\bigcup_{k=0}^{m} c_{p_{k}}$,i.e. $K \subset \bigcup_{k=0}^{m} c_{p_{k}}$. Then for each $\epsilon \in\left(0, \min _{0 \leq k \leq m}\left|S\left(A\left(p_{k}\right)\right)\right|\right)$ there exists $\delta \in\left(0, \min _{1 \leq k \leq m} \delta_{p_{k}}\right)$ such that for the corresponding induced matrix measure $\mu^{H_{\delta}^{0}}$ we have that the inequality

$$
\mu^{H_{\delta}^{0}}(A(p))-S(A(p)) \leq \epsilon
$$

holds for every $p \in K$.

Corollary 11. Let the conditions of Theorem 10 hold.

Then there exist $\delta>0$ and a matrix $H_{\delta} \in \mathbb{C}^{n \times n}$, det $H_{\delta} \neq 0$ defined by (3.1), such that $\mu^{H_{\delta}}(A(p))<0$ for every $p \in K$. 
Proof. Taking into account that for $\epsilon \in\left(0, \min _{0 \leq k \leq m}\left|S\left(A\left(p_{k}\right)\right)\right|\right)$ and $\delta \in$ $\left(0, \min _{1 \leq k \leq m} \delta_{p_{k}}\right)$ the inequality (3.8) holds for every $p \in K$, then obviously $\mu^{H_{\delta}^{0}}(A(p))<0$ for every $p \in K$ too.

Theorem 12. Let $\|.\| \in \Upsilon$ be an arbitrary norm, $K \subset \overline{\mathbb{C}_{+}}$be an arbitrary compact set, $A(p)=\left\{a_{k}^{j}(p)\right\}_{k, j=1}^{n}, B(p)=\left\{b_{k}^{j}(p)\right\}_{k, j=1}^{n}$ and the following conditions hold:

1. The functions $a_{k}^{j}, b_{k}^{j} \in C\left(\overline{\mathbb{C}}_{+}, \mathbb{C}\right), 1 \leq k, j \leq n$.

2. $S(A(p))<0$ and $S(B(p))<0$ for every $p \in K$.

Then there exist $\delta>0$ and a matrix $H_{\delta} \in \mathbb{C}^{n \times n}$, $\operatorname{det} H_{\delta} \neq 0$ defined by (3.1), such that $\mu^{H_{\delta}}(A(p))<0$ and $\mu^{H_{\delta}}(B(p))<0$ for every $p \in K$.

Proof. Let $\|.\| \in \Upsilon$ be an arbitrary norm, $p_{0} \in K$ be an arbitrary fixed point and $H^{0} \in \mathbb{C}^{n \times n}$ be a nonsingular matrix which transforms the matrix $A\left(p_{0}\right)$ in its canonical Jordan form. Let for each $p \in K$ the number $\epsilon_{p} \in\left(0, \min \left(\left|S\left(A\left(p_{0}\right)\right)\right|,|S(A(p))|,|S(B(p))|\right)\right)$ be arbitrary. Then according Theorem 10 there exists $\delta_{p}=\delta_{p}\left(\epsilon_{p},\|\|.\right)>0$ such that for each $\delta \in\left(0, \delta_{p}\right)$ there exists a matrix $H_{\delta_{p}}^{0} \in \mathbb{C}^{n \times n}$ with $\operatorname{det} H_{\delta_{p}}^{0} \neq 0$ for which for the corresponding induced matrix measure $\mu^{H_{\delta_{p}}^{0}}$ all inequalities $\mu^{H_{\delta_{p}}^{0}}\left(A\left(p_{0}\right)\right)<0, \mu^{H_{\delta_{p}}^{0}}(A(p))<0$ and $\mu^{H_{\delta_{p}}^{0}}(B(p))<0$ hold, where the matrix $H_{\delta_{p}}^{0}$ is defined by $(3.1)$, i.e. $H_{\delta_{p}}^{0}=$ $D_{\delta_{p}} H^{0}$ and $D_{\delta_{p}}=\operatorname{diag}\left(1, \delta_{p}^{-1}, \delta_{p}^{-2}, \ldots, \delta_{p}^{-(n-1)}\right)$. The rest of the proof can be completed as the proof of Theorem 10.

Remark 13. It is clear that the assertion of Theorem 12 remains true for arbitrary finite number of matrices $A_{i}(p), i=1,2, \ldots, l, l \in \mathbb{N}$.

\section{Acknowledgments}

This research has been supported by Grant FP17-FMI-008.

\section{References}

[1] G. Dahlquist, Stability and Error Bounds in the Numerical Integration of Ordinary Differential Equations, Diss. 1958; reprinted in Trans. Royal Inst. of Technology, No. 130, Stockholm (1959).

[2] L.Kolev, Eigenvalue range determination for interval and parametric matrices, Int. J. Circ. Theor. Appl. 38, (2010), 1027-1061, doi: 10.1002/cta.609. 
[3] L.Kolev, S. Filipova-Petrakieva, Outer bounds on the eigenvalues of interval matrices, the complex eigenvalues case, Proc. of the Technical University of Sofia, 51, (2001), 139-147.

[4] L. Kolev, Determining the positive definiteness margin of interval matrices, Reliable Computing 13(5) (2007), 445-466, doi: 10.1007/s11155-007-9046-5.

[5] S. M. Lozinskii, Error estimate for numerical integration of ordinary differential equations, Izv. Vyssh. Uchebn. Zaved. Mat., 5 (1958), 52-90.

[6] O. Pastravanu, M. Matcovschi, Stability of matrix polytopes with a dominant vertex and implications for system dynamics, Abstract and Applied Analysis, Article ID 396759 (2013), doi: 10.1155/2013/396759.

[7] O. Pastravanu, M. Matcovschi, Sufficient conditions for Schur and Hurwitz diagonal stability of complex interval matrices, Linear Algebra Appl., 467 (2015), 149-173, doi: 10.1016/j.laa.2014.11.005.

[8] O. Pastravanu, M. Voicu, Generalized matrix diagonal stability and linear dynamical systems, Linear Algebra Appl., 419(2) (2006) 299-310, doi: 10.1016/j.laa.2006.04.021.

[9] T. Strom, On logarithmic norms, SIAM J. Numer. Anal., 12 (1975) 741-753.

[10] Z. Zahreddine, Matrix measure and application to stability of matrices and interval dynamical systems, International Journal of Mathematics and Mathematical Sciences, 2 (2003), 75-85, doi: 10.1155/S0161171203202295. 